\title{
Effect of antiviral therapy on the outcomes of mechanically ventilated patients with herpes simplex virus detected in the respiratory tract: a systematic review and meta-analysis
}

\author{
Stefan Hagel ${ }^{1 *}$ D, André Scherag ${ }^{2,3}$, Lukas Schuierer ${ }^{4,5}$, Reinhard Hoffmann ${ }^{5}$, Charles-Edouard Luyt ${ }^{6}$,
} Mathias W. Pletz , Miriam Kesselmeier, ${ }^{2,3+}$ and Sebastian Weis ${ }^{1,7,8+}$

\begin{abstract}
Background: Herpes simplex virus (HSV) is frequently detected in the respiratory tract of mechanically ventilated patients. The aim of this study was to assess current evidence to determine whether antiviral therapy is associated with better outcomes in these patients.

Methods: MEDLINE, ISI Web of Science, Cochrane Database and ClinicalTrials.gov were searched from inception to 25 May 2020. All clinical studies investigating the effects of antiviral therapy on the outcome of mechanically ventilated ICU patients in whom HSV was detected in the respiratory tract were eligible for inclusion, regardless of study design, publication status or language. Titles and abstracts were reviewed independently by two authors. If the articles seemed eligible, full-text articles were reviewed and data extracted.

We performed a random-effects meta-analysis to estimate relative risks (RRs) with corresponding 95\% confidence intervals (Cls). The primary endpoint was hospital all-cause mortality.

Results: Nine studies were included in the meta-analysis (one randomized controlled trial, eight cohort studies). Antiviral treatment was associated with lower hospital mortality (with antiviral treatment, 40.6\% (189 out of 465 patients); without, 52.7\% (193 out of 366 patients); RR 0.74 [0.64, 0.85]; eight studies, low quality of evidence). Furthermore, antiviral treatment was associated with lower 30-day mortality (RR 0.75 [0.59, 0.94]; three studies, very low quality of evidence). We did not observe evidence for differences in ICU mortality (RR 0.73 [0.51, 1.05]; three studies, very low quality of evidence).
\end{abstract}

\footnotetext{
* Correspondence: stefan.hagel@med.uni-jena.de

${ }^{\dagger}$ Miriam Kesselmeier and Sebastian Weis contributed equally to this work. ${ }^{1}$ Institute for Infectious Diseases and Infection Control, Jena University Hospital - Friedrich Schiller University Jena, Jena, Germany

Full list of author information is available at the end of the article
}

C C The Author(s). 2020 Open Access This article is licensed under a Creative Commons Attribution 4.0 International License, which permits use, sharing, adaptation, distribution and reproduction in any medium or format, as long as you give appropriate credit to the original author(s) and the source, provide a link to the Creative Commons licence, and indicate if changes were made. The images or other third party material in this article are included in the article's Creative Commons licence, unless indicated otherwise in a credit line to the material. If material is not included in the article's Creative Commons licence and your intended use is not permitted by statutory regulation or exceeds the permitted use, you will need to obtain permission directly from the copyright holder. To view a copy of this licence, visit http://creativecommons.org/licenses/by/4.0/ The Creative Commons Public Domain Dedication waiver (http://creativecommons.org/publicdomain/zero/1.0/) applies to the data made available in this article, unless otherwise stated in a credit line to the data. 
(Continued from previous page)

Conclusions: This meta-analysis of the available data shows that antiviral therapy might result in lower hospital and 30-day all-cause mortality in mechanically ventilated ICU patients who are positive for HSV in the respiratory tract. However, this result must be interpreted with great caution due to the high risk of bias and limited number of patients. Large, well-designed randomized controlled clinical trials are urgently needed.

Trial registration: The study was registered in advance on International Prospective Register of Systematic Reviews (CRD42020180053).

Keywords: Herpes simplex, Mechanical ventilation, Antiviral therapy, Critically ill

\section{Introduction}

Herpes simplex virus (HSV) causes a variety of infections that affect mucocutaneous surfaces, the central nervous system and, occasionally, visceral organs. After primary infection, HSV invades neurons and subsequently remains in a non-replicating form in the sensory ganglia for the entire lifespan of the infected individual [1]. During the latency period, reactivation of the infection can be triggered by a wide range of stimuli, including local (e.g. tissue lesions or UV light) or systemic stimuli (e.g. fever, impairment of the immune system during critical illness or surgery) [1]. Oropharyngeal HSV reactivation has been shown to occur in 20 to $54 \%$ of critically ill patients, depending on the study population and the severity of disease [2-6]. In patients with prolonged mechanical ventilation, HSV can be detected in the bronchoalveolar lavage (BAL) in up to $64 \%$ of patients in intensive care units (ICUs) [7]. However, whether antiviral therapy improves patients' outcomes in these circumstances is a matter of debate $[8,9]$. It remains unclear whether the detection of HSV represents harmless viral shedding as a consequence of reactivation, reflecting the severity of the underlying disease and/or a surrogate for a state of decreased virological immune response (immunoparalysis), or a true clinical infection requiring antiviral therapy [911]. Study results are conflicting. Mortality in patients with HSV in respiratory secretions was increased in some $[6,12-14]$ but not in other studies $[5,7,15]$. The decision is further complicated by the challenge of rendering a confident clinical diagnosis of HSV bronchopulmonary infection in these patients [16]. The aim of the present systematic review and meta-analysis is to assess the current evidence as to whether antiviral therapy is associated with better outcomes in mechanically ventilated ICU patients in whom HSV was detected in the respiratory tract.

\section{Material and methods}

The systematic review and meta-analysis were performed according to the Preferred Reporting Items for Systematic Reviews and Meta-Analyses (PRISMA) and Meta-Analysis of Observational Studies in Epidemiology (MOOSE) guidelines. Complete details, including electronic search strategy, objectives, criteria for study selection, eligibility, data collection and assessment of study quality, were registered in advance in the PROSPERO International Prospective Register of Systematic Reviews (CRD42020180053).

\section{Literature search and data extraction}

All clinical studies investigating the effects of antiviral therapy on the outcome of mechanically ventilated ICU patients in whom HSV was detected in the respiratory tract were eligible for inclusion, regardless of study design, publication status or language. Information on our primary outcome (in-hospital all-cause mortality) and our secondary outcomes (30-day all-cause mortality, ICU all-cause mortality) must have been reported. There was no minimal number of patients. There were no minimal numbers of exclusion criteria. We searched $M E D L$ INE, ISI Web of Science (Science Citation Index Expanded), Cochrane Database and ClinicalTrials.gov from inception to 25 May 2020 for eligible clinical studies. The search terms are provided (Supplementary Table 1). We complemented the database searches by screening the reference lists of relevant studies and reviews as well as by directly asking selected experts for studies that they were aware of but were not already included in this analysis. Two authors (S.W., S.H.) independently performed the literature search, identified all studies potentially relevant for this review and selected studies that were included. Conflicts over inclusion were resolved through consensus. All study authors were contacted in order to retrieve all additional available data (including information on missing data).

\section{Data extraction and risk of bias assessment}

One author (S.H.) extracted the number of patients and events for both treatment groups (with or without antiviral therapy). A second author (S.W.) independently validated the results. One study [17] reported mortality; however, it did not specify the timepoint. We decided to include this article and classified it as hospital all-cause mortality. To assess potential heterogeneity of the study populations, we extracted information on study designs and settings as well as summarized patient characteristics. Two authors (S.W. and S.H.) independently performed a 
formal risk assessment of the individual studies according to the Newcastle-Ottawa Scale (NOS) (Supplementary Table 2) [18]. Differences in judgement were resolved by discussion. The certainty of evidence of the individual studies was judged according to the guidelines of the Grading of Recommendations Assessment, Development and Evaluation (GRADE) working group $[19,20]$.

\section{Synthesis of results}

We applied random-effects meta-analyses to estimate relative risks (RRs) for the primary and secondary endpoints. Studies were pooled according to the MantelHaenszel and DerSimonian-Laird methods for withinstudy and between-study variance, respectively [21-23]. We applied a continuity correction of 0.5 in studies with cell frequencies of zero. Statistical heterogeneity was evaluated by the $I^{2}$ statistic. Heterogeneity was judged accordingly: 0 to $40 \%=$ low, 30 to $60 \%=$ moderate, 50 to $90 \%=$ substantial (or high) and 75 to $100 \%=$ consider able. The importance of this measure depends on the magnitude and direction of effects as well as the precision of the estimate (often judged by the corresponding $p$ value from the chi-squared test) [24]. To identify potential evidence of publication bias, we additionally inspected funnel plots. For the sensitivity analyses, we (i) performed the meta-analysis with odds ratios (ORs) as a measure of effect size, (ii) applied leave-one-out crossvalidation and (iii) assumed that mortality reported in [17] was ICU all-cause mortality. We reported point estimates (RR or OR) together with their corresponding 95\% confidence intervals (CIs) and presented the results as forest plots. All analyses were performed with R (version 3.6.0; R package meta, version 4.11.0) [25].

\section{Results}

\section{Study selection}

Our database search revealed 884 reports (see Table 1, Fig. 1). In addition, we considered one of our own studies, which was unpublished at that point [27], as well as seven studies from other sources. We removed $145 \mathrm{du}$ plicates. Of the remaining 747 references, 738 studies were excluded due to lack of relevant information regarding our predefined outcome parameters. Finally, we identified and analysed nine studies comprising 1069 patients who had investigated at least one of the outcome parameters. All but one of these studies were nonrandomized cohort studies, with the majority of retrospective design. Among the included studies, acyclovir was most often used for therapy. The only randomized study was performed by Luyt et al. [28]. In this doubleblind, placebo-controlled trial, 238 patients who received mechanical ventilation for at least $96 \mathrm{~h}$ and continued to receive mechanical ventilation for at least $48 \mathrm{~h}$ with HSV oropharyngeal reactivation were included. The aim of the study was to determine whether pre-emptive treatment with intravenous acyclovir reduces the duration of mechanical ventilation in patients with HSV oropharyngeal reactivation. Detailed characteristics of the included studies are provided in Table 1.

\section{Hospital all-cause mortality}

For the primary endpoint, hospital all-cause mortality, data from eight studies comprising 831 patients were used (Fig. 2a) [5, 7, 11, 17, 26, 27, 29, 31]. In these studies, hospital all-cause mortality was lower in patients with antiviral therapy (40.6\%, 189 out of 465 patients) than in patients without antiviral therapy (52.7\%, 193 out of 366 patients). This resulted in a RR of 0.74 [0.64, $0.85]$. Heterogeneity was low in the studies reporting hospital all-cause mortality $\left(I^{2}=0 \%, p=0.43\right)$. These results were supported by the sensitivity analyses (Supplementary Fig. 1A, Supplementary Table 3).

\section{Secondary outcomes}

Overall, we identified three studies [27-29] reporting 30-day all-cause mortality in 633 patients (Fig. 2b) and four studies $[11,27,29,31]$ reporting ICU all-cause mortality in 629 patients (Fig. 2c). Pooling these studies, antiviral treatment was associated with lower 30-day all-cause mortality (RR 0.75 $[0.59,0.94])$. However, no evidence for an association with ICU all-cause mortality was observed (RR $0.73[0.51,1.05]$ ). Heterogeneity was low in 30-day all-cause mortality $\left(I^{2}=\right.$ $17 \%, p=0.30$ ) but substantial in ICU all-cause mortality $\left(I^{2}=68 \%, p=0.03\right)$. These results were in line with those of the sensitivity analyses (Supplementary Fig. $1 \mathrm{~B}$ and C, Supplementary Table 3; ICU all-cause mortality including [17]: RR $0.75[0.57,1.00], I^{2}=56.5 \%$ with $p=0.06$ ).

\section{Assessment of bias}

Funnel plots revealed no evidence of publication bias, particularly for those studies reporting hospital all-cause mortality (Supplementary Fig. 2). However, the small number of studies reporting the secondary endpoints hampered the assessment. According to the GRADE classification, we judged the quality of evidence to be low for hospital all-cause mortality and very low for the secondary endpoints due to the inherent high risk of bias introduced by retrospective, non-randomized trial designs (Table 2). In particular, indication bias could not be ruled out, which could possibly over- or underestimate the favourable treatment effect of antiviral therapy (i.e. patients with a higher probability of survival are more likely to be treated and vice versa). Subcategories of bias (such as selection, allocation, performance, attrition or reporting bias) were not assessed. 


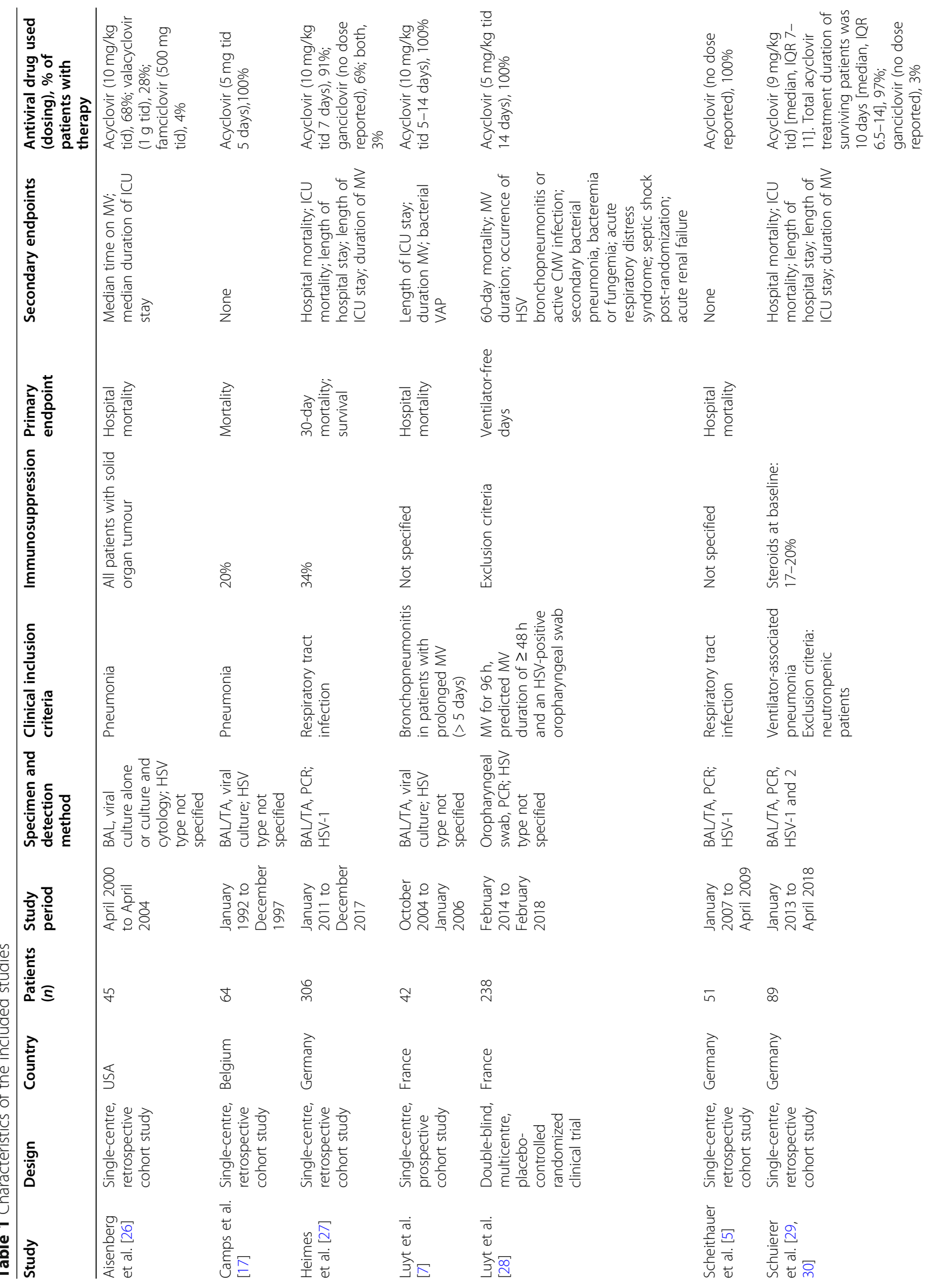




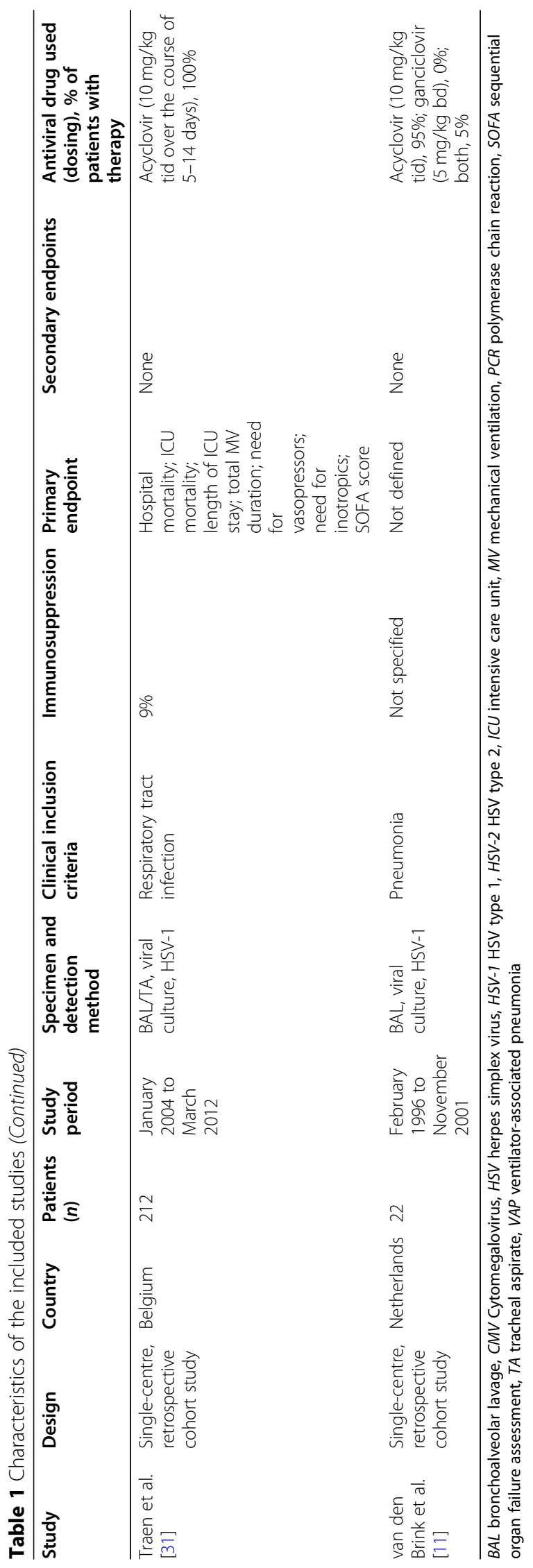




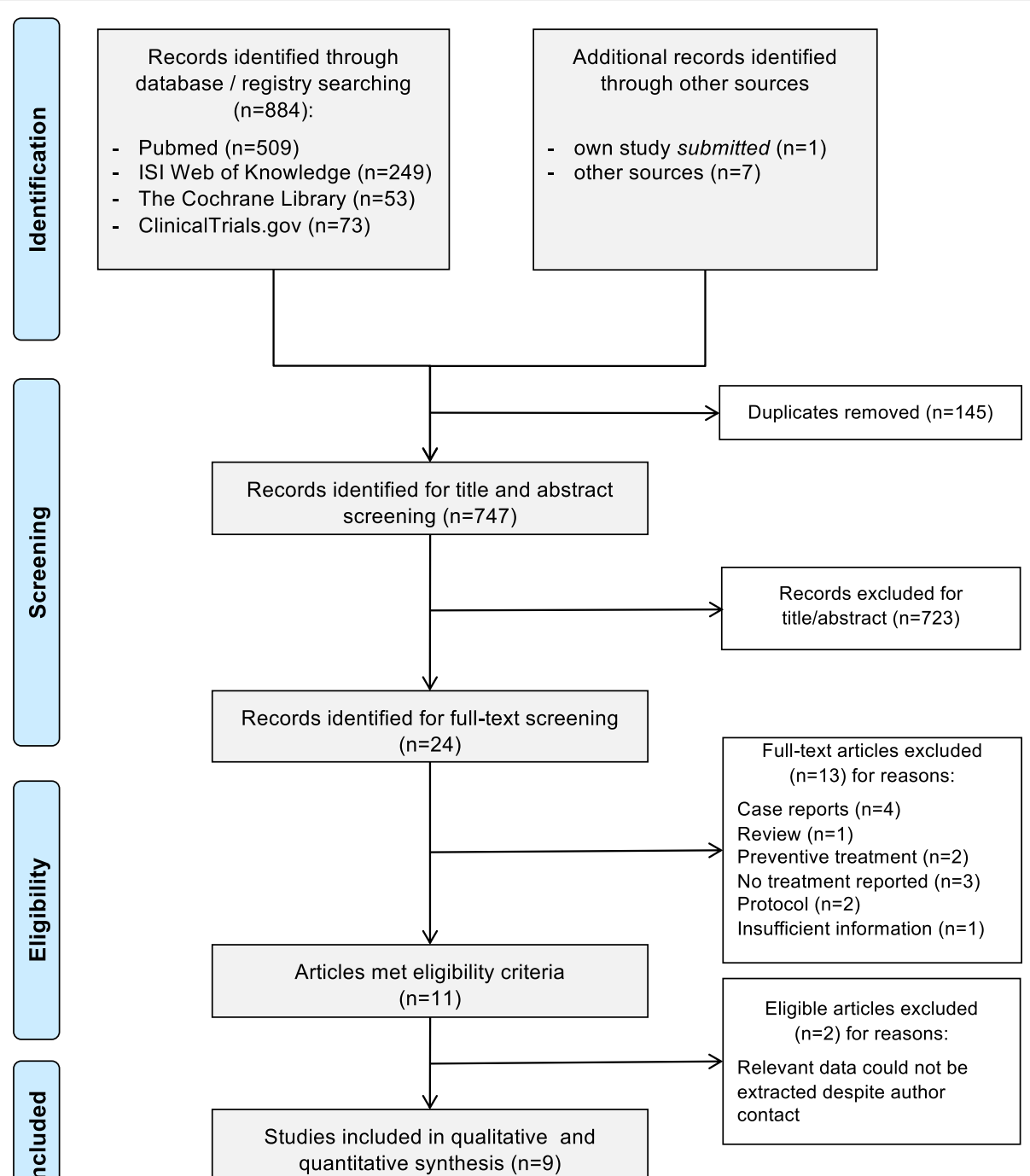

Fig. 1 PRISMA flow diagram of study identification and selection process for outcome analysis

\section{Discussion}

To the best of our knowledge, this is the first systematic review and meta-analysis aiming to summarize the current evidence for antiviral therapy for mechanically ventilated ICU patients in whom HSV was detected in the respiratory tract. Our literature search identified nine studies with 1069 patients overall. The results of the metaanalysis of the available data showed that antiviral therapy might improve hospital all-cause mortality as well as 30day all-cause mortality in mechanically ventilated patients in whom HSV was detected in the respiratory tract. These results suggest that the detection of HSV in these circumstances is of clinical relevance, albeit most likely only in some cases, not all. This is supported by an observation from Luyt et al. [7]. In that retrospective study, in $32.6 \%$ of 129 patients in whom HSV was detected in the BAL, HSV bronchopneumonitis was histologically confirmed.
However, due to its complexity and risks, a lung biopsy for securing a diagnosis of HSV bronchopneumonitis is not routinely feasible in critically ill patients. In addition, cytologic changes typical for HSV infection, i.e. multinucleated giant cells with specific nuclear inclusions, are admittedly specific but suffer from poor sensitivity [32]. On the other hand, rendering a confident clinical diagnosis of HSV bronchopulmonary infection in mechanically ventilated patients without lung biopsy is challenging. Clinical symptoms of HSV bronchopneumonitis are nonspecific and often mimic bacterial pneumonia, with fever, hypoxemia and purulent pulmonary secretions. The same is true for the radiologic examinations, which are often nonspecific and can show ground-glass attenuations, air-space consolidations and interlobular thickening [16].

Randomized controlled studies are necessary to identify patients who could benefit (the most) from antiviral 
A Hospital all-cause mortality

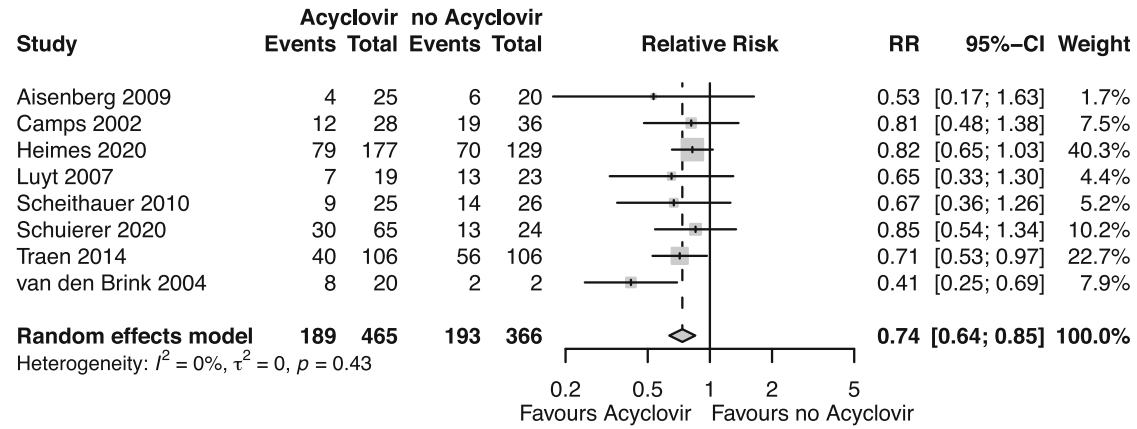

B 30-day all-cause mortality

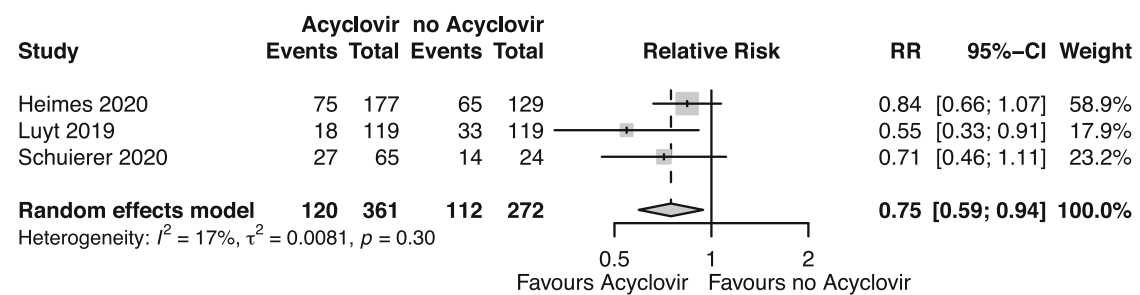

\section{ICU all-cause mortality}

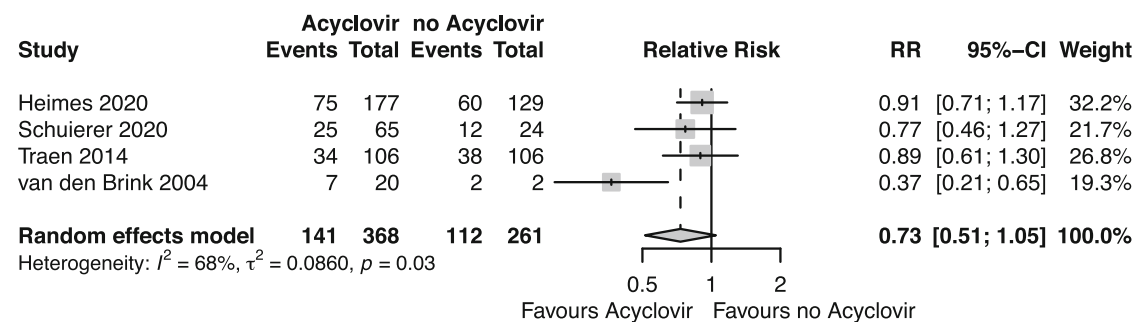

Fig. 2 Results for the primary and secondary endpoints in mechanically ventilated patients with HSV detection in respiratory tract. Cl, confidence interval; HSV, herpes simplex virus; ICU, intensive care unit; RR, relative risk

therapy and to reduce therapy-associated adverse events in others. In addition to clinical signs of a respiratory tract infection, possible criteria might be location of detection, i.e. upper respiratory tract/oropharyngeal cavity versus lower respiratory tract/BAL [31] and amount of virus load [29]. For example, Traen et al. [31] retrospectively analysed 212 ICU patients with a positive HSV-1 culture from the endotracheal/bronchial aspirate $(n=$ $162)$ or BAL $(n=50)$. In their study, using propensity score matching, acyclovir therapy was associated with lower ICU mortality (OR $0.31,95 \%$ CI $0.18-0.56$ ) and lower in-hospital mortality (OR $0.28,95 \%$ CI $0.17-0.46$ ). In particular, the subgroup of patients with HSV-1 detected in the BAL accounted mostly for this difference. Most recently, in a retrospective study, Schuierer et al. [29] investigated whether patients with ventilatorassociated pneumonia (VAP) not responding to antibiotics and in whom HSV could be detected in respiratory secretions (BAL or tracheal aspirates) would benefit from acyclovir treatment. In their cohort of 425 patients screened for HSV type 1 or 2, $57(13.4 \%)$ patients had a low viral load $\left(10^{3}-10^{5} \mathrm{HSV}\right.$ copies $\left./ \mathrm{ml}\right)$ and $69(16.2 \%)$ patients a high $\left(>10^{5} \mathrm{HSV}\right.$ copies $\left./ \mathrm{ml}\right)$ viral load. Thirty patients (7\%) with a low viral load and 59 (14\%) patients with a high viral load fulfilled the strict inclusion criteria, i.e. VAP not responding to antibiotics, and were included in the analyses. The authors observed in patients with a high viral load that acyclovir therapy was associated with lower hazard rates for ICU death (treated, 20 out 49 patients died; untreated, 6 out of 10 died; hazard ratio (HR) $0.31,95 \% \mathrm{CI} 0.11-0.92$ ) as well as for 30-day mortality (treated, 21 out of 49 patients died; untreated, 8 out of 10; HR $0.32,95 \%$ CI $0.12-0.85$ ) and resulted in better circulatory and pulmonary oxygenation function over the course of acyclovir treatment compared to no acyclovir treatment $[29,30]$. Thus, material from the 


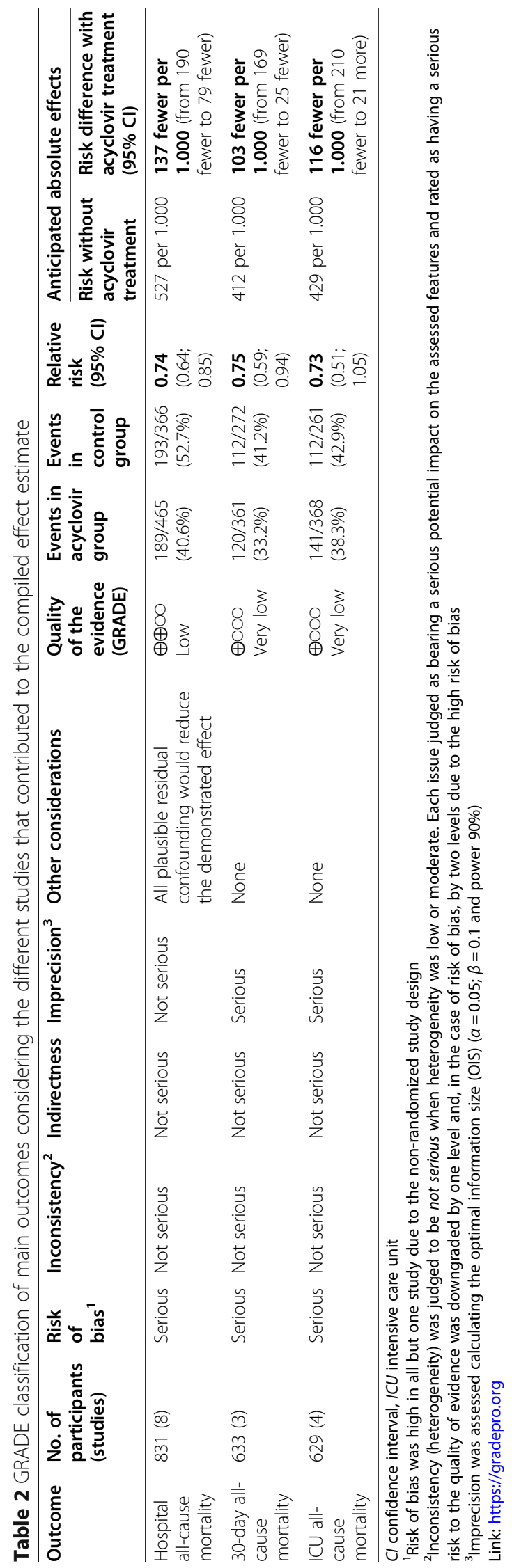


lower respiratory tract and evaluation of viral load in combination with clinical signs and high likelihood of viral pneumonia might be helpful for identifying patients who may benefit the most from antiviral therapy. However, the possibility of adverse events related to antiviral therapy has also to be taken into account. Nephrotoxicity is the most important side effect of acyclovir, with an overall incidence of acute kidney injury (AKI) of 13\%, half of which are KDIGO grade $2 / 3$, as recently reported by Ryan et al. [33]. However, studies showed that acyclovir-associated nephrotoxicity was usually reversible and could be minimized by slow infusion and adequate hydration [34]. Moreover, three of the studies included in this meta-analysis reporting nephrotoxicity did not observe a significant deterioration of renal function [27-30].

\section{Limitations}

The findings and interpretations of this meta-analysis and systematic review are limited by the quality of available evidence. The majority of available evidence was derived from non-randomized, single-centre studies with an inherent high risk of bias. In addition, studies were heterogeneous in terms of included and analysed study cohorts, ranging from patients with HSV detection in the oropharyngeal cavity only [28] to patients with histologically confirmed HSV bronchopneumonitis [7]. To account for this heterogeneity, we used random-effects meta-analyses and performed sensitivity analyses that supported the results of our main analyses. Finally, we did not manually search for unpublished studies, other than conference proceedings that are covered by the utilized electronic databases. Notwithstanding these limitations, the present meta-analysis provides the most comprehensive evaluation of the evidence for antiviral therapy for mechanically ventilated ICU patients in whom HSV was detected in the respiratory tract.

\section{Conclusion}

HSV is frequently detected in the respiratory tract of mechanically ventilated ICU patients. Our meta-analysis of the available data suggests that antiviral therapy is associated with lower hospital all-cause mortality as well as 30-day all-cause mortality. Multicentre, randomized controlled studies are urgently required to identify patients who may benefit the most from antiviral therapy.

\section{Supplementary information}

Supplementary information accompanies this paper at https://doi.org/10. 1186/s13054-020-03296-5.

Additional file 1: Supplementary Table 1. Literature search terms. Supplementary Table 2. Newcastle-Ottawa Scale (NOS) assessing the quality of the individual studies included in the meta-analysis.
Footnotes: ${ }^{1}$ The main outcome was in-hospital overall mortality. ${ }^{2}$ Only a few were patients included. Comparability cannot be assessed. Selection. 1) Representativeness of the exposed cohort: a) truly representative of intensive care patients*, b) somewhat representative of the average intensive care patient ${ }^{*}$, c) selective group of patients at the ICU only, d) no description of the derivation of the cohort. 2) Selection of the nonexposed cohort: a) drawn from the same population as the exposed cohort *, b) drawn from a different source, c) no description of the derivation of the non-exposed cohort. 3) Ascertainment of exposure: a) secure record and rated as appropriate if diagnosis was based on PCR results or culture from bronchoalveolar lavage or swaps* b) structured interview, c) written self-report, d) no descriptio. 4) Demonstration that outcome of interest was not present at start of study: a) yes *, b) no. Comparabilit. 1) Comparability of cohorts on the basis of the design or analysis; a) study controls for the most important factor, such as age, gender, comorbidities * b) ** Rated as appropriate with ** when propensity score matching or a prospective randomized trial was performed. Outcome. 1) Assessment of outcome; a) independent blind assessment *, b) record linkage *, c) self-reported, d) no descriptio. 2) Was follow-up long enough for outcomes to occur? a) yes (in hospital mortality) *, b) no. 3) Adequacy of follow up of cohorts, a) complete follow up - all subjects accounted for *, b) subjects lost to follow up unlikely to introduce bias - small number lost - > 20\%*, c) Kaplan-Meier Plot Curve provided *, d) follow up rate < 20\% and no description of those lost, e) no statement. Supplementary Table 3 Results of leave-one-out cross-validation meta-analyses for the primary and secondary endpoints in herpes simplex virus (HSV) patients, comparing those who were treated with acyclovir to those who were not treated with antiviral drug. The pooled relative risk estimate (RR) and its 95\% confidence interval $(\mathrm{Cl})$ as well as the heterogeneity statistic $\mathrm{I}^{2}$ were considered when omitting the indicated study. Abbreviations: ICU, intensive care unit.

Additional file 2: Supplementary Figure 1. Results for the primary and the secondary endpoints in mechanically ventilated patients with HSV detection in respiratory tract (measure of effect size: odds ratio). Abbreviations: Cl, confidence interval; HSV, herpes simplex virus; ICU, intensive care unit; $\mathrm{OR}$, odds ratio.

Additional file 3: Supplementary Figure 2. Assessment of publication bias for the primary and secondary endpoints. In the funnel plot, the individual study results are represented as grey points, and the pooled estimate is indicated by a dotted line. Abbreviations: ICU, intensive care unit.

\section{Acknowledgements}

Not applicable

\section{Authors' contributions}

S.H. and S.W. contributed equally to the study design, study selection, data extraction, quality assessment and writing of the manuscript. M.K. and A.S. performed the data analysis and contributed in writing and revising the manuscript. C.E.L., L.S., M.P. and R.H. contributed in writing and revising the manuscript for important intellectual content. All authors approved the final manuscript.

\section{Funding}

M.K., A.S. and S.W. were supported by the Integrated Research and Treatment Center-Center for Sepsis Control and Care (CSCC) at the Jena University Hospital, funded by the German Ministry of Education and Research (BMBF No. 01EO1502). Open Access funding enabled and organized by Projekt DEAL.

Availability of data and materials

All data generated or analysed during this study are included in this published article.

Ethics approval and consent to participate Not applicable

Consent for publication Not applicable 


\section{Competing interests}

C.E.L. has received personal fees from Carmat, Merck, Biomérieux, Thermofischer Brahms, Bayer Healthcare and Faron, outside the submitted work. All other authors declare that they have no potential financial conflicts of interest regarding the contents of the publication.

\section{Author details \\ ${ }^{1}$ Institute for Infectious Diseases and Infection Control, Jena University Hospital - Friedrich Schiller University Jena, Jena, Germany. ${ }^{2}$ Research Group Clinical Epidemiology, Center for Sepsis Control and Care (CSCC), Jena University Hospital - Friedrich Schiller University Jena, Jena, Germany. ${ }^{3}$ Institute of Medical Statistics, Computer and Data Sciences, Jena University Hospital - Friedrich Schiller University Jena, Jena, Germany. ${ }^{4}$ TUM Graduate School, Technical University of Munich (TUM), Munich, Germany. Institute for Laboratory Medicine and Microbiology, University Hospital Augsburg, Augsburg, Germany. ${ }^{6}$ Service de Médecine Intensive Réanimation, Institut de Cardiologie, Groupe Hospitalier Pitié-Salpêtrière, Sorbonne-Université, Assistance Publique Hôpitaux de Paris, Paris, France. ${ }^{7}$ Department of Anesthesiology and Intensive Care Therapy, Jena University Hospital - Friedrich Schiller University Jena, Jena, Germany. ${ }^{8}$ Center for Sepsis Control and Care, Jena University Hospital - Friedrich Schiller University Jena, Jena, Germany.}

Received: 14 July 2020 Accepted: 16 September 2020 Published online: 29 September 2020

\section{References}

1. Simoons-Smit AM, Kraan EM, Beishuizen A, Strack van Schijndel RJ, Vandenbroucke-Grauls CM. Herpes simplex virus type 1 and respiratory disease in critically-ill patients: real pathogen or innocent bystander? Clin Microbiol Infect. 2006;12:1050-9.

2. Tuxen DV, Wilson JW, Cade JF. Prevention of lower respiratory herpes simplex virus infection with acyclovir in patients with the adult respiratory distress syndrome. Am Rev Respir Dis. 1987;136:402-5.

3. Saugel B, Jakobus J, Huber W, Hoffmann D, Holzapfel K, Protzer U, et al. Herpes simplex virus in bronchoalveolar lavage fluid of medical intensive care unit patients: association with lung injury and outcome. J Crit Care. 2016;32:138-44

4. Lepiller Q, Sueur C, Solis M, Barth H, Glady L, Lefebvre F, et al. Clinical relevance of herpes simplex virus viremia in intensive care unit patients. J Inf Secur. 2015;71:93-100.

5. Scheithauer S, Manemann AK, Krüger S, Häusler M, Krüttgen A, Lemmen SW, et al. Impact of herpes simplex virus detection in respiratory specimens of patients with suspected viral pneumonia. Infection. 2010;38:401-5.

6. Linssen CFM, Jacobs JA, Stelma FF, van Mook WNKA, Terporten P, Vink C, et al. Herpes simplex virus load in bronchoalveolar lavage fluid is related to poor outcome in critically ill patients. Intensive Care Med. 2008;34:2202-9.

7. Luyt C-E, Combes A, Deback C, Aubriot-Lorton M-H, Nieszkowska A, Trouillet J$L$, et al. Herpes simplex virus lung infection in patients undergoing prolonged mechanical ventilation. Am J Respir Crit Care Med. 2007;175:935-42.

8. Chanques G, Jaber S. Treating HSV and CMV reactivations in critically ill patients who are not immunocompromised: con. Intensive Care Med. 2014; 40:1950-3.

9. Forel J-M, Martin-Loeches I, Luyt C-E. Treating HSV and CMV reactivations in critically ill patients who are not immunocompromised: pro. Intensive Care Med. 2014;40:1945-9.

10. De Vos N, Van Hoovels L, Vankeerberghen A, Van Vaerenbergh K, Boel A, Demeyer l, et al. Monitoring of herpes simplex virus in the lower respiratory tract of critically ill patients using real-time PCR: a prospective study. Clin Microbiol Infect. 2009;15:358-63.

11. van den Brink J-W, Simoons-Smit AM, Beishuizen A, Girbes ARJ, van Schijndel RJM S, ABJ G. Respiratory herpes simplex virus type 1 infection/colonisation in the critically ill: marker or mediator? J Clin Virol. 2004;30:68-72.

12. Brenner T, Rosenhagen C, Hornig I, Schmidt K, Lichtenstern C, Mieth M, et al. Viral infections in septic shock (VISS-trial)-crosslinks between inflammation and immunosuppression. J Surg Res. 2012;176:571-82.

13. Stram MN, Suciu CN, Seheult JN, McCullough MA, Kader M, Wells A, et al. Herpes simplex virus-1 qPCR in the diagnosis of lower respiratory tract infections in organ transplant recipients and critically ill patients. Am J Clin Pathol. 2018;150:522-32.
14. Bouza E, Giannella M, Torres MV, Catalán P, Sánchez-Carrillo C, Hernandez RI, et al. Herpes simplex virus: a marker of severity in bacterial ventilatorassociated pneumonia. J Crit Care. 2011;26:432 e1-6.

15. Assink-de Jong E, Groeneveld ABJ, Pettersson AM, Koek A, VandenbrouckeGrauls CMJE, Beishuizen A, et al. Clinical correlates of herpes simplex virus type 1 loads in the lower respiratory tract of critically ill patients. J Clin Virol. 2013;58:79-83.

16. Chong S, Kim TS, Cho EY. Herpes simplex virus pneumonia: high-resolution CT findings. Br J Radiol. 2010;83:585-9.

17. Camps K, Jorens PG, Demey HE, Pattyn SR, leven M. Clinical significance of herpes simplex virus in the lower respiratory tract of critically ill patients. Eur J Clin Microbiol Infect Dis. 2002;21:758-9.

18. Wells G, Shea B, O'Connell D, Peterson J, Welch V, Losos M. The Newcastle-Ottawa Scale (NOS) for assessing the quality of nonrandomised studies in meta-analyses. [cited 2020 Mar 31]. Available from: http://www. ohri.ca/programs/clinical_epidemiology/oxford.asp.

19. Andrews J, Guyatt G, Oxman AD, Alderson P, Dahm P, Falck-Ytter Y, et al. GRADE guidelines: 14. Going from evidence to recommendations: the significance and presentation of recommendations. J Clin Epidemiol. 2013; 66:719-25.

20. Andrews JC, Schünemann HJ, Oxman AD, Pottie K, Meerpohl JJ, Coello PA, et al. GRADE guidelines: 15. Going from evidence to recommendationdeterminants of a recommendation's direction and strength. J Clin Epidemiol. 2013;66:726-35.

21. Robins J, Breslow N, Greenland S. Estimators of the Mantel-Haenszel variance consistent in both sparse data and large-strata limiting models. Biometrics. 1986;42:311-23.

22. Greenland S, Robins JM. Estimation of a common effect parameter from sparse follow-up data. Biometrics. 1985;41:55-68.

23. DerSimonian R, Laird N. Meta-analysis in clinical trials. Control Clin Trials. 1986;7:177-88.

24. Higgins GS. Cochrane Handbook for Systematic Reviews of In- terventions Version 5.1.0 [updated March 2011] The Cochrane collaboration. Available from: http://www.cochrane-handbook.org.

25. Schwarzer G. An R package for meta-analysis. R News. 2007;7:40-5.

26. Aisenberg GM, Aisenberg G, Torres HA, Torres H, Tarrand J, Safdar A, et al. Herpes simplex virus lower respiratory tract infection in patients with solid tumors. Cancer. 2009;115:199-206.

27. Heimes E, Baier M, Forstner C, Weis S, Bauer M, Fritzenwanger M, et al. Effect of antiviral therapy on the outcome of mechanically ventilated patients with herpes simplex virus type 1 in bronchoalveolar lavage fluid: a retrospective cohort study. Chest. 2020. https://doi.org/10.1016/j.chest.2020. 06.056.

28. Luyt C-E, Forel J-M, Hajage D, Jaber S, Cayot-Constantin S, Rimmelé T, et al. Acyclovir for mechanically ventilated patients with herpes simplex virus oropharyngeal reactivation: a randomized clinical trial. JAMA Intern Med. 2020; 180(2):263-72. https://doi.org/10.1001/jamainternmed.2019.5713.

29. Schuierer L, Gebhard M, Ruf H-G, Jaschinski U, Berghaus TM, Wittmann M, et al. Impact of acyclovir use on survival of patients with ventilatorassociated pneumonia and high load herpes simplex virus replication. Crit Care. 2020;24:12

30. Honore PM, Mugisha A, Kugener L, Redant S, Attou R, Gallerani A, et al. Acyclovir for ventilator-associated pneumonia refractory to antibiotics and with high viral herpes simplex load: we are not sure. Crit Care. 2020;24:262.

31. Traen $S$, Bochanen $N$, leven $M$, Schepens $T$, Bruynseels $P$, Verbrugghe $W$, et al. Is acyclovir effective among critically ill patients with herpes simplex in the respiratory tract? J Clin Virol. 2014;60:215-21.

32. Byers RJ, Hasleton PS, Quigley A, Dennett C, Klapper PE, Cleator GM, et al. Pulmonary herpes simplex in burns patients. Eur Respir J. 1996;9:2313-7.

33. Ryan L, Heed A, Foster J, Valappil M, Schmid ML, Duncan CJA. Acute kidney injury (AKI) associated with intravenous aciclovir in adults: incidence and risk factors in clinical practice. Int J Infect Dis. 2018;74:97-9.

34. Whitley RJ, Gnann JW. Acyclovir: a decade later. N Engl J Med. 1992;327: 782-9.

\section{Publisher's Note}

Springer Nature remains neutral with regard to jurisdictional claims in published maps and institutional affiliations. 